\title{
SERIES WHOSE PRODUCT IS ABSOLUTELY CONVERGENT.
}

BY PROFESSOR FLORIAN CAJORI.

(Read before Section A of the American Association for the Advancement of Science, Pittsburg, July 1, 1902.)

$\S 1$. That two absolutely convergent series yield an absolutely convergent product was first shown by Cauchy.* About three quarters of a century later Alfred Pringsheim pointed out that an absolutely convergent product may result also from the multiplication of a conditionally convergent or even a divergent series by an absolutely convergent series. $\dagger$ That the product of two conditionally convergent series, or of a conditionally convergent series and a divergent series, or of two divergent series, may be absolutely convergent was first made public by the present writer. $\$$ Thereupon Alfred Pringsheim treated the subject from a more general point of view and, by very simple methods, showed that the property in question is typical of certain classes of series. $\S$ The present writer developed a new class of series possessing this property, demonstrated the validity of the fundamental laws of algebra in the multiplication of infinite series, and generalized a theorem of Abel on the multiplication of series. $\|$ In the present article we aim to generalize some of the results previously obtained relating to absolutely convergent products of two or more series.

$\S 2$. In this investigation we shall start with an absolutely convergent series and determine pairs of series which are factors of the assumed series. Given the absolutely convergent series

in which

$$
U \equiv \sum_{n=0}^{n=\infty} u_{n}
$$

$$
u_{n} \equiv \sum_{r=0}^{r=n} c_{r} e_{r} c_{n-r}^{\prime} e_{n-r}
$$

* Analyse Algébrique, 1821 , page 147.

†Muth. Annalen, vol. 21 (1883), pp 357-359.

$\ddagger$ Transactions of the American Mathematiral Soriety, vol. 2, pp 25-36, January 1901; Srience, new series, vol. 14, p. 395 September 13, 1901.

\$ Transmations of the American Mathematical Society, vol. 2, pp. 404-412, October, 1901.

|| Bulletin, 2d series, vol. 8, pages 231-236, March, 1902. 
$\boldsymbol{e}_{r}$ is the general term of any absolutely convergent series, say

$$
e_{r} \equiv \frac{1}{(z+r)[\log (z+r)]^{\lambda}},
$$

$\lambda>1$, and $c_{r}, c_{r}^{\prime}$ are constants either real or complex. That series (1) is absolutely convergent becomes evident, if we observe that it is the product formed according to Cauchy's multiplication rule, of the two absolutely convergent series

Let us assume

$$
\sum_{r=0}^{r=\infty} c_{r} e_{r} \quad \text { and } \quad \sum_{r=0}^{r=\infty} c_{r}^{\prime} e_{r} .
$$

$$
\begin{gathered}
a_{r-s}-a_{r}=c_{r} e_{r}, \\
h_{r}\left(b_{r-t}-b_{r}\right)=c_{r}^{\prime} e_{r},
\end{gathered}
$$

where $s$ and $t$ are positive integers, $a$ and $b$ are real or complex numbers and $h_{r}$ is an odd or an even power of -1 . Let it be agreed that $a$ and $b$ cannot have negative subscripts; in other words, that $a_{-x}=b_{-x}=0$. We have

$$
u_{n} \equiv \sum_{r=0}^{r=n} c_{r} e_{r} \cdot c_{n-r}^{\prime} e_{n-r}=\sum_{r=0}^{r=n} h_{n-r}\left(a_{r-s}-a_{r}\right)\left(b_{n-r-t}-b_{n-r}\right) .
$$

If we perform the indicated multiplications and collect the coefficients of $a_{r}$, we obtain

$$
u_{n} \equiv \sum_{r=0}^{r=n} a_{r}\left(-h_{n-r} b_{n-r-t}+h_{n-r} b_{n-r}+h_{n-r-s} b_{n-r-s-t}\right.
$$

If we assume $h_{x}=-h_{x+t}$, we have

$$
\left.-h_{n-r-s} b_{n-r-s}\right) \text {. }
$$

$$
u_{n} \equiv \sum_{r=0}^{r=n}\left(a_{r-t}+a_{r}\right)\left(h_{n-r} b_{n-r}-h_{n-r-s} b_{n-r-\imath}\right) .
$$

It will be noticed that if, in the two terms in (3) which involve the factors $a_{r}$ and $a_{r-t}$, respectively, we remove the parentheses, we obtain eight terms which are distributed among three terms 
of the series in (4), namely the three terms which involve, respectively, the parentheses

$$
\left(a_{r-2 t}+a_{r-t}\right), \quad\left(a_{r-t}+a_{r}\right), \quad\left(a_{r}+a_{r+t}\right) .
$$

From the inspection of series (4) we readily see that the series (1) may be considered to be the product of the following two series :

$$
\begin{aligned}
\sum_{r=0}^{r=\infty}\left(a_{r-t}+a_{r}\right) \equiv a_{0}+\alpha_{1}+\cdots+ & a_{t-1}+\left(a_{0}+a_{t}\right) \\
& +\left(a_{1}+a_{t+1}\right)+\cdots
\end{aligned}
$$

and

$$
\begin{aligned}
\sum_{r=0}^{r=\infty}\left(h_{r} b_{r}-h_{r-s} b_{r-8}\right) \equiv h_{0} b_{0}+h_{1} b_{1} & +\cdots+h_{s-1} b_{s-1} \\
& +\left(h_{s} b_{s}-h_{0} b_{0}\right)+\cdots .
\end{aligned}
$$

According to the condition $h_{r}=-h_{r+t}$, we are permitted to choose any sign we please for any $t$ consecutive factors $h_{r}$. After such a choice has been made, the signs represented by any of the other factors $h_{x}$ are determined.

Since relations (2) are the only conditions imposed upon the values of $a$ and $b$, it is possible to choose these values so that each of the series (5) and (6) is absolutely convergent, conditionally convergent, or divergent. Thus, if $\left|a_{r}\right|$ is of the order of magnitude $e_{r}$, series (5) is absolutely convergent; if

$$
\left|a_{r-t}+a_{r}\right| \geqq \frac{1}{(r+2) \log (r+2)},
$$

but approaches the limit zero as $r$ increases indefinitely and if $\left(a_{r-t}+a_{r}\right)$ is opposite in sign to and has greater numerical value than $\left(a_{r-t+1}+a_{r+1}\right)$, then (5) is conditionally convergent; if $\left|a_{r-t}+a_{r}\right|$ does not approach the limit zero for all values of $r$, as $r$ increases indefinitely, the series (5) is divergent. Similarly for (6). Yet in every case, the product of (5) and (6) is absolutely convergent.

Since, so far as we know, no two divergent series with complex terms have before been given, whose product is absolutely convergent, it may be well to construct a special example. Let 
$a_{r}=b_{r}=A+B_{r} e_{r}$, where $A$ and $B_{r}$ are complex constants. Moreover, let $s \stackrel{r}{=} 1$ and $t=2, h_{0}=-h_{1}=+1$. It will be seen that values which are not infinite can be assigned to the coefficients $c_{r}$ and $c_{r}^{\prime}$ so that equations (2) are satisfied. By substitution in the two series (5) and (6) we obtain the following two series :

$$
\left.\begin{array}{r}
S_{1} \equiv\left(A+B_{0} e_{0}\right)+\left(A+B_{1} e_{1}\right)+\left(2 A+B_{2} e_{2}+B_{0} e_{0}\right) \\
+\left(2 A+B_{3} e_{3}+B_{1} e_{1}\right)+\cdots, \\
S_{2} \equiv\left(A+B_{0} e_{0}\right)-\left(2 A+B_{1} e_{1}+B_{0} e_{0}\right)-\left(B_{2} e_{2}-B_{1} e_{1}\right) \\
+\left(2 A+B_{3} e_{3}+B_{2} e_{2}\right)+\left(B_{4} e_{4}-B_{3} e_{3}\right)-\cdots .
\end{array}\right\}
$$

It will be seen that both series in (7) are divergent and complex, and that their product is absolutely convergent. Another pair of complex series possessing this property is given at the close of this article.

Since all the terms in the series $S_{1}$ are preceded by the positive sign, it is readily seen that any positive integral power of $S_{1}$ is a divergent series whose terms increase numerically without limit as $r$ increases without limit. The same conclusion holds for the series $S_{2}$. Since $S_{1} \cdots S_{2} \cdots S_{1} \cdots S_{2} \cdots$ [to $p$ pairs of factors] $=\left(S_{1} S_{2}\right)\left(S_{1} S_{2}\right) \cdots$ [to $p$ parentheses] $=S_{1}^{p} \cdots S_{2}^{p}$, and since $S_{1} \cdots S_{2}$ is an absolutely convergent complex product, it follows that the product of the two complex series $S_{1}^{p}$ and $S_{2}^{p}$, the terms of both of which increase numerically without limit as $r$ increases without limit, is absolutely convergent, no matter how large a value the integer $p$ may have.*

If we let $a_{r}$ and $b_{r}$ be positive and decreasing monotonously toward zero in such a way that $\Sigma a_{r}$ and $\Sigma b_{r}$ are both divergent, if moreover, $t=2, s=1, h_{0}=+1, h_{1}=-1$, then (5) and (6) reduce to the two following series, one divergent, the other conditionally convergent, given by Pringsheim : $\uparrow$

$$
\sum_{r=0}^{r=\infty}\left(a_{r-2}+a_{r}\right) \text { and } \sum_{r=0}^{r=\infty}(-1)^{r+\left[\frac{r-1}{2}\right]}\left(b_{r-1}+(-1)^{r-1} b_{r}\right) .
$$

* See Bulletin, 2d series, vol. 8 (1902), pp. 233-236.

† See Transactions of the American Mathem 'itica? Society, vol. 2, p. 408, equation (B). The notation $\left[\begin{array}{l}r-1 \\ -2\end{array}\right]$ signifies here the largest integer contained in $\frac{r-1}{2}$. 
In order to deduce from (5) and (6) the pair of conditionally convergent series, whose product is absolutely convergent, which we gave in the Bulletis, volume 8 (1902), page 231, let $s=t=4 ; h_{0}=h_{1}=h_{2}=h_{3}=+1 ; r=4 v, 4 v+1,4 v+2$, or $4 v+3 ; r^{\prime}=4 v$. Let moreover the parenthesis $\left(a_{r-t}+a_{r}\right)$, which we represent for convenience by $a_{r}^{\prime}$, be a real number which is positive when $r=4 v$ or $4 v+1$ and negative when $r=4 v+2$ or $4 v+3$, and such that $\left|a_{r}^{\prime}\right| \leqq r^{-u}$, where $\frac{1}{2}<u \leqq 1$, and $\Sigma\left|\alpha_{r}^{\prime}\right|$ is divergent. Without violating conditions (2) we may assume further

$$
\begin{gathered}
a_{r^{\prime}}^{\prime}=a_{r^{\prime}+1}^{\prime}, \quad\left|a_{r^{\prime}+2}^{\prime}\right|-a_{r^{\prime}}^{\prime}=e_{r^{\prime}} \pm w_{r^{\prime}}, \\
a_{r^{\prime}+2}^{\prime}=a_{r^{\prime}+3}^{\prime}, \quad\left|a_{r^{\prime}+2}^{\prime}\right|-a_{r^{\prime}+4}^{\prime}=e_{r^{\prime}+4} \pm w_{r^{\prime}+4},
\end{gathered}
$$

where $w_{r}$ is a quantity numerically not greater than

$$
\frac{1}{(2+r)^{2-u}[\log (2+r)]^{\lambda}}
$$

Similarly we may assume, without violating conditions (2),

$$
h_{r} b_{r}-h_{r-s} b_{r-s} \equiv b_{r}^{\prime}, \quad\left|b_{r}^{\prime}\right| \leqq r^{-u}, \quad \sum_{r=0}^{r=\infty}\left|b_{r}^{\prime}\right| \text { divergent }
$$

$b_{r}^{\prime}$ a real number which is positive when $r=4 v$ and $4 v+2$, and negative when $r=4 v+1$ and $4 v+3$; also

$$
\begin{array}{cl}
b_{r^{\prime}}^{\prime}=b_{r^{\prime}+2}^{\prime}, & b_{r^{\prime}}^{\prime}-\left|b_{r^{\prime}+1}^{\prime}\right|=e_{r^{\prime}} \pm w_{r^{\prime}} \\
b_{r^{\prime}+1}^{\prime}=b_{r^{\prime}+3}^{\prime}, & b_{r^{\prime}+4}^{\prime}-\left|b_{r^{\prime}+1}^{\prime}\right|=e_{r^{\prime}+4} \pm w_{r^{\prime}+4^{*}}
\end{array}
$$

There result from these assumptions the two conditionally convergent series $\sum_{r=0}^{r=\infty} a_{r}^{\prime}$ and $\sum_{r=0}^{r=\infty} b_{r}^{\prime}$, whose product is an absolutely convergent series, which were previously given by us in the last mentioned article.

$\S 7$. If we are given a series

$$
\sum_{r=0}^{r=\infty}\left(a_{r-t}+a_{r}\right)
$$


such that $a_{r-s}-a_{r}=c_{r} e_{r}$, it is quite evident that we can always find a mate for it, such that the product of the two shall be absolutely convergent.

Again it is possible to find two series, every term in the product of which, after the $(t+1)$ th term, vanishes identically. Moreover, the two series may be so chosen that the product possesses the additional property of having for its sum any desired finite number $N$. To bring this about modify conditions (2) thus,

$$
a_{r \rightarrow s}-a_{r}=c d^{r}
$$

where $d$ is any number, and, for $r \geqq t$,

$$
h_{r}\left(b_{r-t}-b_{r}\right)=0 \text {. }
$$

Then $\sum_{r=0}^{r=\infty} u_{r}$ is still absolutely convergent. Let $t$ be even and

$$
\begin{aligned}
& b_{t-1}=d b_{t-2}=d^{2} b_{t-3}=\cdots=d^{t-1} b_{0}, \\
& h_{0}=-h_{1}=h_{2}=-h_{3}=\cdots=-h_{t}=1 .
\end{aligned}
$$

Then we have, for $r \geqq t$,

$u_{r}=b_{0} c d^{r}-b_{0} d \cdot c d^{r-1}+b_{0} d^{2} \cdot c d^{r-2}-\cdots-b_{0} d^{t-1} \cdot c d^{r-t+1} \equiv 0$.

Under the above conditions the two series (5) and (6) have a product, every term of which, after the $(t+1)$ th term, vanishes identically.

To make the sum of the product of the two series (5) and (6) equal to $N$, place the sum of the first $t+1$ terms in the product equal to $N$ and then determine the values of $d$ which satisfy this condition.

Thus, let $t=2, s=1, c=+1$, then from $a_{r-1}-a_{r}=d^{2}$, for $r \geqq 1$, we get the series (5) (the first term of which is assumed to be $a_{0}$ )

$$
\begin{aligned}
a_{0}+\left(a_{0}-d\right)+\left(2 a_{0}-d-d^{2}\right)+\left(2 a_{0}-2 d-d^{2}-d^{3}\right) \\
+\left(2 a_{0}-2 d-2 d^{2}-d^{3}-d^{4}\right)+\cdots
\end{aligned}
$$

Assuming $b_{0}=1$, the series (6) becomes

$$
\begin{aligned}
1-(d+1)+(d-1)+(d+1)-(d-1) & -(d+1) \\
& +(d-1)+\cdots .
\end{aligned}
$$


All the terms in the product of these two series, after the second term, vanish. Putting the sum of the first two terms $=N=0$, we have $a_{0}-d\left(a_{0}+1\right)=0$. If we assume $a_{0}=-\frac{10}{9}$, then $d=10$, and the two factor series become

$$
\begin{aligned}
U & =-\frac{10}{9}-11 \frac{1}{9}-112 \frac{2}{9}-\cdots \\
U^{\prime} & =1-11+9+11-9-11+9+11-\cdots
\end{aligned}
$$

Since $U \cdot U^{\prime}=0$, we have $U^{p} \cdot U^{\prime p}=0$. As all the terms in $U$ are of the same sign, it is easily seen that $U^{p}$ is divergent for all positive integral values of $p . \quad U^{\prime}$ and $U^{\prime p}$ are also divergent.

$\S 8$. If we assume $t=2, s=1, c=-1, a_{0}=1, b_{0}=1$, $N=0$, then the condition that the sum of the product of $(5)$ and (6) shall vanish becomes $d^{2}+1=0$ and (letting $i=\sqrt{-1}$ ) the factor series thus obtained are the two complex divergent series

$$
\begin{aligned}
& 1+(1+i)+i+0+1+(i+1)+i+0+\cdots \\
& 1-(1+i)+(i-1)+(i+1)-(i-1)-(i+1)+\cdots
\end{aligned}
$$

Colorado College, Colorado Springis, April 12, 1902 .

\section{THREE SETS OF GENERATIONAL RELATIONS DEFINING THE ABSTRACT SIMPLE GROUP OF ORDER 504.}

BY PROFESSOR L. E. DICKSON.

(Read before the American Mathematical Society, October 25, 1902.)

1. Considerable interest attaches to the simple group of order 504. The existence of this simple group was discovered by Professor Cole.* This was one of the facts that lead Professor Moore $\dagger$ to his investigation of the linear fractional group in the general Galois field, resulting in the discovery of a new doubly infinite system of simple groups. 1893.

* "On a certain simple group," Mathematical Papers, Chicago Congress of + Buxletris, December, 1893 ; Mathematical Papers, Congress of 1893. 